\title{
Quantum Rotor Approach to the Mott-Insulator Transition in the Bose-Hubbard Model
}

\author{
T.P. POLAK \\ Faculty of Physics, Adam Mickiewicz University of Poznań \\ Umultowska 85, 61-614 Poznań, Poland \\ AND T.K. Kopé́ \\ Institute for Low Temperatures and Structure Research \\ Polish Academy of Sciences \\ P.O.B. 1410, 50-950 Wrocław 2, Poland \\ We present the novel approach to the Bose-Hubbard model using the \\ $\mathrm{U}(1)$ quantum rotor description. We formulate a problem in the phase only \\ action using an effective action formalism and obtain analytical formulae for \\ the critical lines. We show that the nontrivial U(1) phase field configurations \\ have an impact on the phase diagrams. The topological character of the \\ quantum field is governed by terms of the integer charges - winding numbers \\ $n$. The comparison of the presented results with recently obtained quantum \\ Monte Carlo numerical calculations suggests that the competition between \\ quantum effects in strongly interacting boson systems is correctly captured
} by our model.

PACS numbers: 05.30.Jp, 03.75.Lm, 03.75.Nt

\section{Introduction}

A collection of interacting bosons on a lattice is described by the BoseHubbard (BH) [1] model which captures the physics arising from the competition between kinetic energy of bosons and their on-site interaction. More recently, it has been realized that the Bose-Hubbard model can also be applied to bosons trapped in so-called optical lattices $[2,3]$, where the on-site interaction $U$ can be tuned by the Feshbach resonance and nearest neighbors hopping $t$ by the intensity of the laser beams which form the optical lattice. The coarse graining [4], mean-field theories [5], strong-coupling expansion [6], have been successfully applied to these systems in one- [7], two- [8] and three-dimensional lattices [9]. The progress comes from better computer resources and more efficient algorithm allows to use the quantum Monte Carlo (QMC) method for studies of the BH 
systems $[10,11]$. Optical lattices offer remarkably clean access to a particular Hamiltonian and thereby serve as a model system for testing fundamental theoretical concepts and providing exemplar of quantum many-body effects [12]. It is well known that the ground state of a system of repulsively interacting bosons in a periodic potential can be either in a superfluid state $(\mathrm{SF})$ or in a Mott-insulating state (MI), characterized by integer boson densities. Because the phase of the order parameter and the particle number as conjugate variables are subject to the uncertainty principle $\Delta \phi \Delta n \sim \hbar[13]$ and the bosons can either be in the eigenstate of particle number or phase. The eigenstate of phase is a superfluid and that of particle number is a localized Mott insulator. Therefore, the quantum MI-SF phase transition takes place as the particle density is shifted thus facilitating emergence of the superfluid from the Mott insulating state. The aim of this paper is to extend the mean-field approach for the Bose-Hubbard model in a way to include particle number fluctuations effects and make the qualitative phase diagrams in two and three dimensions more quantitative. The key point of presented approach is to consider the representation of strongly interacting bosons as particles with attached "flux tubes". This introduces a conjugate U(1) phase variable, which acquires dynamic significance from the boson-boson interaction. To facilitate this task we employ the functional integral formulation of the theory that enables us to perform the functional integration over fields defined on different topologically equivalent classes of the U(1) group, i.e., with different winding numbers. Finally, we compare our results for systems at zero temperature with the outcome of the numerical simulations and found a very good agreement for the quantitative results regarding the behavior as we go from the superfluid phase to the Mott insulating phase. The outline of the paper is as follows. In Sect. 2 we introduce the model Hamiltonian and in Sect. 3 we derive an effective U(1) action in the quantum rotor representation. The aim of Sect. 4 is the presentation of the resulting phase diagrams for two- and three-dimensional Bose-Hubbard systems. Finally, Sect. 5 summarizes our results and sets the outlook.

\section{Model Hamiltonian}

We investigate the generic model for the Mott-insulator transition the BoseHubbard model

$$
\mathcal{H}=\frac{U}{2} \sum_{i} n_{i}^{2}-\sum_{\langle i, j\rangle} t_{i j} a_{i}^{\dagger} a_{j}-\bar{\mu} \sum_{i} n_{i},
$$

where $a_{i}^{\dagger}$ and $a_{j}$ stands for the bosonic creation and annihilation operators that obey the canonical commutation relations $\left[a_{i}, a_{j}^{\dagger}\right]=\delta_{i j}, n_{i}=a_{i}^{\dagger} a_{i}$ is the boson number operator on the site $i, U>0$ is the on-site repulsion and $\bar{\mu} / U=\mu / U+1 / 2$ is the shifted reduced chemical potential which controls the number of bosons. Here, $\langle i, j\rangle$ identifies summation over the nearest-neighbor sites. Furthermore, $t_{i j}$ is the hopping matrix element with the dispersion for the bipartite lattice 


$$
t_{\boldsymbol{k}}=2 t \sum_{l=1}^{d} \cos k_{l}
$$

in $d$ dimensions. In this paper we investigate the phase transitions in simple cubic and square lattice.

\section{Method}

\subsection{Decoupling of the Coulomb interaction}

We will adopt the method of the quantum rotor model, developed by one of us [14], to the BH Hamiltonian. The partition function of the system could be written in the usual form

$$
\mathcal{Z}=\int[\mathcal{D} \bar{a} \mathcal{D} a] \mathrm{e}^{-\mathcal{S}[\bar{a}, a]}
$$

where the bosonic path-integral is taken over the complex fields $a_{i}(\tau)$ with the action $\mathcal{S}$ given by

$$
\mathcal{S}=\mathcal{S}_{\mathrm{B}}[\bar{a}, a]+\int_{0}^{\beta} \mathrm{d} \tau \mathcal{H}(\tau),
$$

where

$$
\mathcal{S}_{\mathrm{B}}[\bar{a}, a]=\sum_{i} \int_{0}^{\beta} \mathrm{d} \tau \bar{a}_{i}(\tau) \frac{\partial}{\partial \tau} a_{i}(\tau)
$$

Unfortunately, Hamiltonian is not quadratic in $a_{i}$ and we have to decouple first - the Coulomb term in Eq. (1) by a Gaussian integration over the auxiliary fields $V_{i}(\tau)$. Moreover, we split the field for static $V_{i}^{\mathrm{S}}(\tau)$ and periodic function $V_{i}^{\mathrm{P}}(\tau)$ and introduce the scalar potential field which couples to the local particle number through the Josephson-like relation $\dot{\phi}_{i}(\tau)=V_{i}^{\mathrm{P}}(\tau)$, where the phase field satisfies the periodicity condition $\phi_{i}(\beta)=\phi_{i}(0)$ as a consequence of the periodic properties of the $V_{i}^{\mathrm{P}}(\tau)$ field.

\subsection{Gauge transformation}

We perform the local gauge transformation to the new bosonic variables

$$
\left[\begin{array}{c}
a_{i}(\tau) \\
\bar{a}_{i}(\tau)
\end{array}\right]=\left[\begin{array}{cc}
\mathrm{e}^{\mathrm{i} \phi_{i}(\tau)} & 0 \\
0 & \mathrm{e}^{-\mathrm{i} \phi_{i}(\tau)}
\end{array}\right]\left[\begin{array}{c}
b_{i}(\tau) \\
\bar{b}_{i}(\tau)
\end{array}\right]
$$

The chief merit of the transformation in Eq. (6) is that we have managed to cast the strongly correlated bosonic problem into a system of weakly interacting bosons, submerged in the bath of strongly fluctuating $\mathrm{U}(1)$ gauge potentials (on the high energy scale set by $U$ ). Furthermore, the path-integral includes a summation over winding numbers

$$
\int[\mathcal{D} \phi] \ldots \equiv \sum_{\left\{n_{i}\right\}} \int_{0}^{2 \pi} \prod_{i} \mathrm{~d} \phi_{i}(0) \int_{\phi_{i}(0)}^{\phi(\tau)_{i}+2 \pi n_{i}} \prod_{i} \mathrm{~d} \phi_{i}(\tau) \ldots
$$

and should be performed taking phase configurations that satisfy boundary condition $\phi_{i}(\beta)-\phi_{i}(0)=2 \pi n_{i}$, where $n_{i}$ is integer. 


\subsection{The partition function expressed in the phase fields variables}

We parametrize the boson fields $b_{i}(\tau)=b_{0}+b_{i}^{\prime}(\tau)$ and restrict our calculations to the phase fluctuations dropping the amplitude dependence. From expansion of the trace of the logarithm we take only second term. Finally, action is expressed only in the phase fields variable

$$
\begin{aligned}
\mathcal{S}_{\mathrm{ph}}[\phi] & =\int_{0}^{\beta} \mathrm{d} \tau\left\{\sum_{i}\left[\frac{1}{2 U} \dot{\phi}_{i}^{2}(\tau)+\frac{1}{i} \frac{\bar{\mu}}{U} \dot{\phi}_{i}(\tau)\right]\right. \\
& \left.-\sum_{i, j} \mathrm{e}^{\phi_{i}(\tau)} J_{i j} \mathcal{I}_{i j} \mathrm{e}^{\phi_{j}(\tau)}\right\},
\end{aligned}
$$

where $\mathcal{I}_{i j}=1$ if $i, j$ are the nearest neighbors and equals zero otherwise.

To proceed we replace the phase degrees of freedom by the complex field $\psi_{i}$ which satisfies the quantum periodic boundary condition $\psi_{i}(\beta)=\psi_{i}(0)$. This can be conveniently done using the Fadeev-Popov method with the Dirac delta functional representation in a way used by Kopeć and José [15].

\section{Mott insulator — superfluid phase transition}

Within the phase coherent superfluid state the order parameter $\Psi_{\mathrm{B}}=$ $\left\langle\exp \left(\mathrm{i} \phi_{i}(\tau)\right)\right\rangle$ in the limit $\beta \rightarrow \infty$ becomes

$$
1-\Psi_{\mathrm{B}}^{2}=\frac{1}{2} \int_{-\infty}^{+\infty} \frac{\rho(\xi) \mathrm{d} \xi}{\sqrt{2 \bar{\xi}\left(2 z \frac{t}{U}+\frac{\mu}{U}+\frac{1}{2}\right) \frac{t}{U}+v^{2}\left(\frac{\mu}{U}\right)}}
$$

with $v(\mu / U)=\operatorname{frac}(\mu / U)-\frac{1}{2}$, where frac $(x)=x-[x]$ is the fractional part of the number and $[x]$ is the floor function which gives the greatest integer less than or equal to $x ; \rho(\xi)$ is the density of states, where $\xi$ is dimensionless parameter, $\bar{\xi} \equiv \xi_{\max }-\xi$ and $\xi_{\max }$ stands for the maximum value of the dispersion spectrum $t(\boldsymbol{k})$. The zero temperature phase diagram of the model calculated from Eq. (9) is given in Fig. 1. We recognize the particle-hole asymmetric - Mott-insulating lobes similar to what was found in the literature $[4,6]$. In the MI phase bosons are incompressible $\partial n_{\mathrm{B}} / \partial \mu=0$ and localized which means that the total energy is minimized when each site is filled with the same number of atoms. Increasing fluctuations in the phase system reduces fluctuations in the boson number on each site according to the Heisenberg uncertainty relation. Crossing the boundary line bosons can move from one lattice site to the next. The order parameter $\Psi_{\mathrm{B}}$ has a non-vanishing value and system exhibits the long-range phase coherence. This is opposite case to the Mott-insulator where phase coherence is lost. We see that the qualitative shape of the lobes is not the same for $2 \mathrm{D}$ and $3 \mathrm{D}$ cases and steeper for the two-dimensional system.

The effects of the fixed boson number $n_{\mathrm{B}}$ in the system defined by $n_{\mathrm{B}}=$ $N^{-1} \sum_{i}\left\langle\bar{a}_{i}(\tau) a_{i}(\tau)\right\rangle$ and the calculations of a phase diagram for interaction problem $t / U \neq 0$ have to include spatial correlations. The result for the boson density 


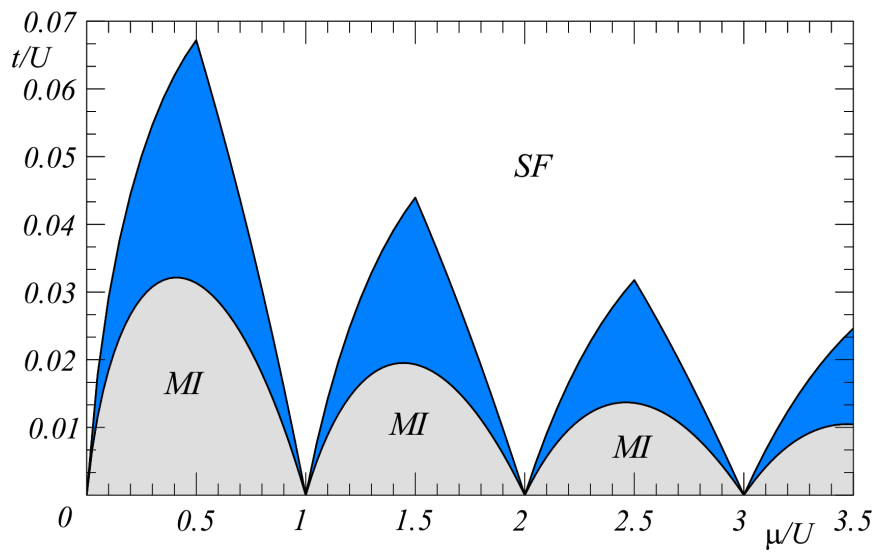

Fig. 1. Phase boundary between the MI and SF phases for square (darker lobes) and cubic lattice (lighter lobes) in the space of the parameters $t / U-\mu / U$.

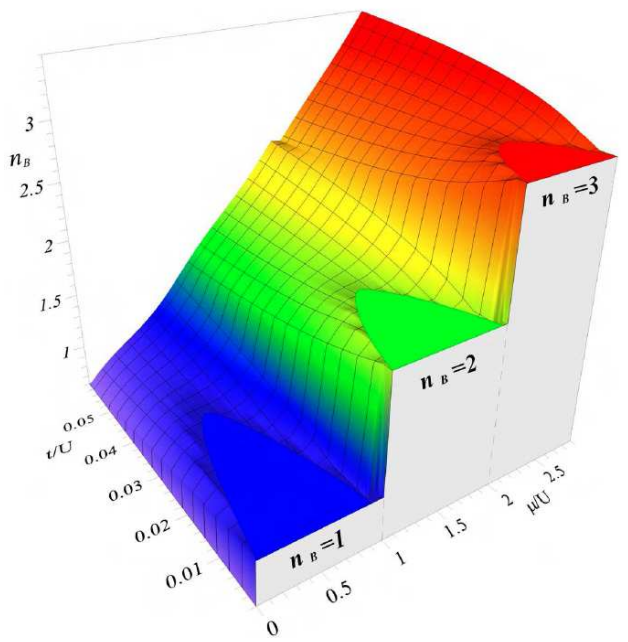

Fig. 2. Boson occupation number $n_{\mathrm{B}}$ at $T=0$ for three-dimensional simple cubic lattice in the space of parameters - chemical potential $\mu / U$ and hopping $t / U$. The Mott insulator is found within each lobe of integer boson density. Inside the first lob on the left the occupation number $n_{\mathrm{B}}$ is equal to one, two, and three in first, second, and third step, respectively.

$n_{\mathrm{B}}$ within the region of superfluidity is given by the expression

$$
n_{\mathrm{B}}=\frac{\mu}{U}+\frac{1}{2}-2 \Psi_{\mathrm{B}}^{2} v\left(\frac{\mu}{U}\right)
$$

where non-vanishing value of the order parameter $\Psi_{\mathrm{B}}$ is calculated from Eq. (9). We see in Fig. 2 that the competition between kinetic and interaction energy is the foundations of the quantum phase transitions in the $\mathrm{BH}$ model. Increasing 
the value of the hopping term (reducing the interaction energy) leads to delocalization of the bosons thus the sharp steps of the MI state become indistinct and in consequence system is superfluid. In Fig. 2 we observe the appearance of the Mott-insulating lobes corresponding to curves from Fig. 1.

\section{Summary and outlook}

In this paper we have presented a study of the Mott-insulator transition of the Bose-Hubbard model. To analyze quantum phase transitions beyond mean-field theory we employed a U(1) quantum rotor approach and a path-integral formulation of quantum mechanics including a summation over a topological charge, explicitly tailored for the $\mathrm{BH}$ Hamiltonian. The effective action formalism allows us to formulate a problem in the phase only action and obtain analytical formulae for the critical lines. We found that our results are in great accordance with the recently published quantum Monte Carlo calculations [11]. Our calculations also improve predictions based on the third-order expansion in $t / U$ that become inaccurate quite far from the tip [6].

\section{Acknowledgments}

One of us (T.K.K.) acknowledges the support by the Ministry of Education and Science MEN under grant No. 1 P03B 10330 in the years 2006-2008.

\section{References}

[1] M.P.A. Fisher, P.B. Weichman, G. Grinstein, D.S. Fisher, Phys. Rev. B 40, 546 (1989).

[2] D. Jaksch, C. Bruder, J.I. Cirac, C.W. Gardiner, P. Zoller, Phys. Rev. Lett. 81, 3108 (1998).

[3] M. Greiner, O. Mandel, T. Esslinger, T.W. Hansch, I. Bloch, Nature 415, 39 (2002).

[4] A.P. Kampf, G.T. Zimanyi, Phys. Rev. B 47, 279 (1993).

[5] K. Sheshadri, H.R. Krishnamurthy, R. Pandit, T.V. Ramakrishnan, Europhys. Lett. 22, 257 (1993).

[6] J.K. Freericks, H. Monien, Phys. Rev. B 53, 2691 (1996).

[7] S.R. Clark, D. Jaksch, Phys. Rev. A 70, 043612 (2004).

[8] M. Niemeyer, J.K. Freericks, H. Monien, Phys. Rev. B 60, 2357 (1999).

[9] A. Sewer, X. Zotos, H. Beck, Phys. Rev. B 66, 140504(R) (2002).

[10] N.V. Prokof'ev, B.V. Svistunov, I.S. Tupitsyn, Phys. Lett. A 238, 253 (1998); J. Exp. Theor. Phys. 87, 310 (1998).

[11] B. Capogrosso-Sansone, N.V. Prokof'ev, B.V. Svistunov, Phys. Rev. B 75, 134302 (2007).

[12] I. Bloch, Nature Phys. 1, 23 (2005).

[13] W.J. Elion, M. Matters, U. Geigenmuller, J.E. Mooij, Nature 371, 594 (2002).

[14] T.K. Kopeć, Phys. Rev. B 70, 054518 (2004).

[15] T.K. Kopeć, J.V. José, Phys. Rev. B 60, 7473 (1999). 